\title{
Aciertos, distorsiones y falacias en la enseñanza de las ciencias naturales de la educación secundaria obligatoria de Argentina
}

\author{
Hector L. Lacreu \\ UNSL - Dpto. Geología \\ lacreu@gmail.com
}

\begin{abstract}
HITS, DISTORTIONS AND FALLACIES IN THE TEACHING OF NATURAL SCIENCES OF COMPULSORY SECONDARY EDUCATION IN ARGENTINA -For the first time in Argentina, was included a curricular space on Earth Sciences. This is a success of the Federal Council of Education (CFE), who adopted the Reference Framework for Oriented Teaching in Natural Science, developed by the Federal Team, although it was not foreseen in the previous regulations. At the same time, other documents on General Formation of Secondary Oriented Education are analyzed, in order to show some pedagogical distortions and fallacies originated by attempting to achieve impossible goals, without the contribution of geology. Both problematic situations are present in recently approved CFE documents, where an epistemological error persists: Geology was omitted as part of the Natural Sciences. This paper includes some requests to the Ministry of Education and proposals for action to solve the problems analyzed. Citation:Lacreu H.L. 2014. Disciplina de Geociências no ensino oficial da Argentina. Terræ Didatica, 10(3):217-226. http://www.ige.unicamp.br/terraedidatica/.
\end{abstract}

KEYWORDS: Teaching Geology, Citizenship Education, Fallacies, Natural Sciences.

RESUMEN Se destaca que en la Argentina, por primera vez, se incluyó un espacio curricular sobre las Ciencias de la Tierra. Se trata de un acierto del Consejo Federal de Educación (CFE), que aprobó el Marco de Referencia para la Formación Orientada en Ciencias Naturales elaborado por el Equipo Federal, pese a que no estaba previsto en las normativas previas. Al mismo tiempo se analizan otros documentos sobre la Formación General de la Educación Secundaria Orientada, a fin de señalar distorsiones pedagógicas y algunas falacias originadas al pretender finalidades imposibles de alcanzar sin el aporte de la Geología. Ambas situaciones problemáticas están presentes en documentos recientemente aprobados, en los que persiste el error epistemológico de omitir la Geología como integrante del campo de las Ciencias Naturales. Se incluyen peticiones al Ministerio de Educación y propuestas de acción para resolver los problemas analizados

PALABRAS CLAVE: Enseñanza de la Geología, Formación Ciudadana, Falacias, Ciencias Naturales. 


\section{Introducción}

El Consejo Federal de Educación (CFE) de la Argentina elaboró los "Lineamientos Políticos y Estratégicos de la Educación Secundaria Obligatoria", promulgados el 15 de octubre de 2009 mediante la Res CFE 84-09. Dicho documento, permite apreciar que la Argentina atraviesa una oportunidad histórica en la que el sistema educativo es objeto de una reforma importante, cuyas finalidades ${ }^{1}$ son por demás alentadoras. En particular, se destaca el énfasis puesto en los sujetos políticos y en su Formación Ciudadana, sin embargo se señala que, para lograr dicha finalidad, los medios propuestos son insuficientes.

Para contextualizar el presente análisis, es necesario realizar dos señalamientos. En primer lugar cabe recordar que en 1994 se incluyeron contenidos de Geociencias en la Enseñanza Obligatoria (Lacreu, 1996, 1997a-b). En los años posteriores, la comunidad geológica universitaria y otros organismos participaron, de diversos modos, en los cursos de capacitación docente implementados por el Ministerio de Educación de la Nación. Sin embargo, ello no fue suficiente, ni eficiente, ya que la llegada de las Geociencias a las aulas fue escasa, por lo que estas temáticas tuvieron escaso arraigo en la cultura de la formación/capacitación de los docentes y por ende en los alumnos.

Además, como veremos más adelante, aún está ausente en la Formación General de la Educación Secundaria Argentina, como se puede ver en los NAP (Núcleos de Aprendizajes Prioritarios), tanto del Ciclo Básico (Res. CFE 249-05), como del Ciclo Orientado (Res CFE 180-12).

Por estas y otras razones, es necesario reflexionar acerca de las causas que impidieron y aún dificultan la enseñanza de la geociencias en la educación obligatoria, así como las causas del paulatino ocultamiento de las geociencias en los documentos oficiales del ministerio de Educación de la Nación durante la década 2003 - 2013. Como parte de dichas reflexiones, cabe interrogarse: ¿Dónde están y quiénes son los actores que supuestamente deberían intervenir para contribuir a la enseñanza de la Geociencias? En ese sentido, y, quizás por la pertenencia del autor se propone una reflexión específica sobre las responsabilidades de la comunidad geológica universitaria en contemplar pasivamente esa situación, pese a las comunicaciones verbales $\mathrm{y}$ a las publicaciones que abordaron y advirtieron

1 Res CFE 84-09 (1), p. 4 sobre dicha necesidad, por ejemplo en la reunión de la AFAG (Asociación de Facultades de Geología, de la República Argentina, realizada el 19/11/1997.

En segundo lugar se señala que la nueva estructura de la Educación Secundaria Obligatoria prevé cuatro ofertas educativas ${ }^{2}$, una de las cuales es la Educación Secundaria Orientada (ESOR) que nos ocupará en este documento. Las otras ofertas son denominadas modalidades (Técnico Profesional, Artística y Educación permanente de jóvenes y adultos)

Dicha ESOR representa un importante avance respecto de la situación preexistente porque de un conjunto de más de 1000 orientaciones del secundario existentes en todo el país, el $\mathrm{CFE}^{3}$ acordó la exclusiva vigencia de 10 (diez) orientaciones, dentro de las cuales, con cierto esfuerzo e imaginación serán reubicadas todas las anteriores. Una de las vigentes es la Orientación en Ciencias Naturales y los egresados obtendrán el título de Bachiller en Cs Naturales.

La ESOR posee una estructura con DOS CICLOS: básico (2-3 años) y orientado (3 años) y con respecto a los saberes a ser enseñados ${ }^{4}$ se establecen DOS CAMPOS: formación general (FG) y formación orientada (FO), que se desarrollarán en 25 horas semanales de clases.

La FG es común a todas las orientaciones y es totalmente compartida en el Ciclo Básico. Sin embargo, su presencia disminuye paulatinamente en el Ciclo Orientado a fin de permitir una presencia creciente de los contenidos de la FO, con los saberes específicos de cada orientación.

En relación con estos campos, cabe señalar que las Ciencias de la Tierra sólo están presentes en la FO de la Orientación en Ciencias Naturales, en consecuencia, los egresados de todas las otras orientaciones carecerán de una formación geocientífica y, por lo tanto, construirán una visión sistémica distorsionada sobre el "funcionamiento" de la naturaleza.

Para finalizar la introducción, se alerta al lector sobre dos aspectos.

En primer lugar, se señala que el autor, desde hace un tiempo, prefiere el término Geología para referirse a la disciplina que debe incluirse en los diseños curriculares de la enseñanza pre universitaria de los países en vías de desarrollo. Sin embargo, en este trabajo también se emplean los términos

2 Res CFE 84-09 (57), p. 15-16

3 Res CFE 84-09 (58), p. 16

4 Res CFE 84-09 (82), p. 20 
Geociencias, Ciencias de la Tierra y en este ámbito se considera que los tres son equivalentes. Tres razones fundamentan la preferencia por el término Geología: 1) En nuestros países, posee un significado tan claro y sencillo como las otras disciplinas de las Ciencias Naturales: biología, física, química y astronomía. 2) No es conveniente profundizar en una descripción científica (cientificista?) exhaustiva sobre las disciplinas a las que pertenecen algunos de los contenidos a ser enseñados. En efecto, los contenidos básicos deben promover la comprensión de los fenómenos geológicos fundamentales (procesos y productos) que están involucrados en el "funcionamiento" de la Tierra y no, necesariamente, el señalamiento de sus pertenencias disciplinares. 3) Desde un punto de vista histórico, los contenidos "Geocientíficos" para la enseñanza preuniversitaria estuvieron y pueden seguir estando dentro del campo "geológico". Ello se comprenderá mejor si se analizan "pro" y "contra" de denominar Ciencias de la Vida o Biociencias a la conocida Biología. Este es, en todo caso, un debate pendiente dentro del campo de Transposición Didáctica, pero que excede el objetivo de esta contribución.

En segundo lugar, debe considerarse que el mencionado CFE (Consejo Federal de Educación) está presidido por el Ministro de Educación de la Nación e integrado por las autoridades responsables de la conducción educativa de cada provincia y tres (3) representantes del Consejo de Universidades. Esta aclaración es necesaria para advertir la importancia nacional que poseen sus resoluciones, así como la gravedad de sus errores. Además se destaca que en dicho organismo participan Rectores, representantes de las universidades. En este caso, existe una responsabilidad adicional, toda vez que en dicho ámbito es inexcusable la responsabilidad de contribuir con la vigilancia epistemológica de los documentos que se promulgan.

\section{Aciertos: las Ciencias de la Tierra en la formación de los bachilleres en Ciencias Naturales}

Es preciso reconocer que, pese a la omisión de las Geociencias en las Resoluciones aprobadas por el CFE, durante el proceso de elaboración de los Marcos de Referencia (Res CFE 84-09-65- p. 17), se incluyeron las Ciencias de la Tierra (Res CFE 142-11-02- p. ¿?) como una de las disciplinas a ser enseñadas, incorporando temáticas geocientíficas de actualidad, con relevancia social.

Como se ha dicho, esta presencia no estaba prevista en las normativas existentes y su inclusión en la Orientación en Ciencias Naturales se produjo a partir del año 2010 por una convergencia de circunstancias derivadas tanto de las convicciones y propuestas pioneras del equipo de Cs. Naturales de la Provincia de Buenos Aires, como del apoyo de los equipos curriculares del Ministerio de Educación de la Nación y del Equipo Federal ad-hoc del que participó el autor.

Actualmente, varias provincias han reformulado sus diseños curriculares para el Bachillerato Orientado e incluyeron la disciplina Geociencias (ver Fig. 1). En efecto, hasta el presente, dicho espacio curricular está claramente definido en las provincias de Buenos Aires, Córdoba y Salta, donde Geociencias está localizada en $5^{\circ}$ ó $3^{\circ}$ año, con una carga horaria de 2 hs ó 3 hs semanales. En el caso de las provincias de Entre Ríos y Misiones, las Geociencias están presentes, pero con menor relevancia curricular.

Es la primera vez que se incluye a las Ciencias de la Tierra como espacio Curricular de un modo similar a las disciplinas con las que tradicionalmente se definía el campo de las Ciencias Naturales. Celebramos esta novedad, aunque lo hacemos críticamente porque el crédito horario es exiguo y aún no se avanzó acerca de los criterios para definir el perfil del docente responsable de esta disciplina ni respecto de un programa que paulatinamente promueva su formación/capacitación.

Además de lo expuesto, se considera necesario recuperar, y completar, los contenidos Geocientíficos incluidos en los Contenidos Básicos Orientados aprobados en 1997 (ver: Res. CFEC 57/97b).

\section{Distorsión en la enseñanza de las Ciencias Naturales}

También, es necesario señalar que en la Educación Secundaria Obligatoria Orientada existen carencias relacionadas con la enseñanza de las Geociencias. Sin embargo, para contextualizar el análisis crítico es necesario mencionar que los contenidos de Geociencias incluidos en los años 1996-7 fueron "diluidos" en las nuevas normativas.

En efecto, los NAP de la Educación Primaria de Argentina (Res CFE 228-04 y 235-05), en el Campo de las Ciencias Naturales se incluye el título "En Relación con la Tierra, el Universo y sus 


\begin{tabular}{|c|c|c|}
\hline BUENOS AIRES & 5to año & $2 \mathrm{hs} / \mathrm{sem}$ \\
\hline \multicolumn{3}{|c|}{$\begin{array}{l}\text { UNIDADES 1. La geósfera y su dinámica } \\
\text { 2. El paisaje geológico } \\
\text { 3. Recursos y riesgos geológicos } \\
\text { 4. Historia geológica del paisaje }\end{array}$} \\
\hline \multicolumn{3}{|c|}{$\begin{array}{l}\text { http://servicios2.abc.gov.ar/lainstitucion/organismos/consejogeneral/disenioscurriculares/secundaria/quinto/ } \\
\text { naturales/marco_naturales.pdf }\end{array}$} \\
\hline CÓRDOBA & 5to año & $3 \mathrm{hs} / \mathrm{sem}$ \\
\hline \multicolumn{3}{|c|}{$\begin{array}{l}\text { EJES: + Estructura, composición y dinámica de la Tierra } \\
\text { + Historia geológica de la Tierra } \\
\text { + Recursos y riesgos en los sistemas terrestres }\end{array}$} \\
\hline \multicolumn{3}{|c|}{$\begin{array}{l}\text { http://www.igualdadycalidadcba.gov.ar/SIPEC-CBA/publicaciones/EducacionSecundaria/LISTO\%20PDF/ } \\
\text { Orientacion\%20CsNat.pdf }\end{array}$} \\
\hline SALTA & 3er año & $2 \mathrm{hs} / \mathrm{sem}$ \\
\hline \multicolumn{3}{|c|}{$\begin{array}{l}\text { EJES: + La geósfera y su dinámica } \\
\quad \text { + El paisaje geológico } \\
\text { + El medio natural: suelo, atmósfera e hidrósfera. }\end{array}$} \\
\hline
\end{tabular}

\begin{tabular}{|l|l|l}
\hline ENTRE RÍOS & 5 to año & $i$
\end{tabular}

Enunciados poco organizados: los conceptos integradores que estructuran el currículo de Ciencias de la Tierra serán: la organización, la diversidad, la interacción y los cambios: La dinámica de la Tierra desde una perspectiva ambiocéntrica. Diferentes modelos del universo, Composición, origen, estructura, función y dinámica las capas de la Tierra: Geósfera, Atmósfera e Hidrósfera, Tectónica de placas, Procesos internos y externos.

http://www.aprender.entrerios.edu.ar/servicios/curriculares/nivel-secundario.htm

Observaciones: En La Formación General, más de una vez se menciona a las Cs de la Tierra, se expone su pertenencia al campo de las Cs Naturales. Sin embargo no aparece en sus diseños curriculares.

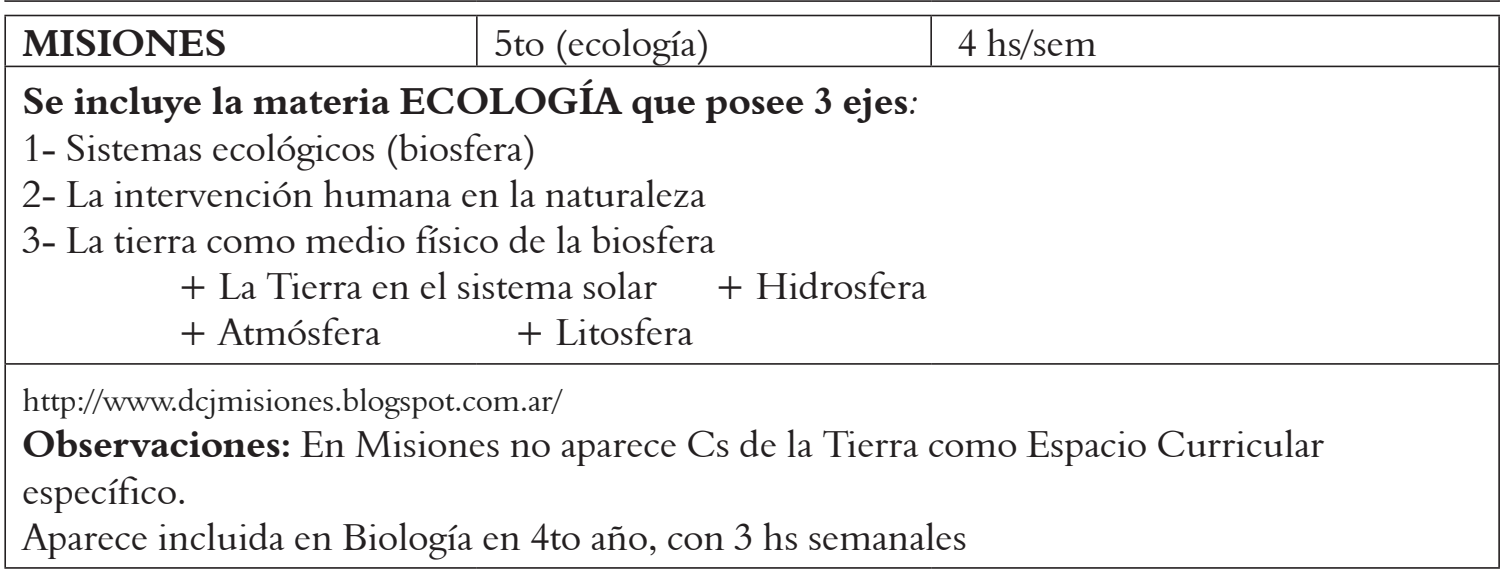

Figura I. Las Geociencias en los diseños curriculares de 5 provincias argentinas (junio 2013): 
Cambios", pero su descripción es deficitaria tanto en sus contenidos como en su secuenciación. Así, las únicas menciones allí efectuadas son: las "geoformas y sus cambios" que está presente sólo en 2do grado (7 años), la "tectónica de placas" se menciona únicamente en 4to grado ( 9 años) y minerales-rocas no son mencionados. Estas carencias también se manifiestan en los "cuadernos para el aula" publicados por el Ministerio de Educación (ME, 2008) como orientación para la enseñanza de las Ciencias Naturales.

Como se podrá advertir, esta temática sobre la educación primaria es muy amplia y requiere un tratamiento específico, sin embargo, este breve relato parece suficiente para concluir que la deficiente formación geocientífica prevista para la Educación Secundaria, no está ni siquiera "compensada" en la formación precedente.

En relación con la Educación Secundaria Orientada, en los NAP del Ciclo Básico (Res. CFE 29405), se puede advertir que el campo de las Ciencias Naturales está presente en todos los años bajo el título: "La Tierra, el universo y sus cambios". Sin embargo, políticamente se ha optado por caracterizar dichos NAP desde una mirada fundamentalmente astronómica, e invisibilizar los contenidos sobre los Recursos Naturales y los Riesgos Ambientales, tal como se enuncia en el documento del Tercer Ciclo de los CBC de la EGB (CFEC 57/97a), aún vigentes.

En dicho documento se expresa claramente que “... se espera que los alumnos al finalizar la Educación Polimodal podrán":

- Identificar y explicar procesos de distintas escalas temporo-espaciales que generan, deterioran, agotan o inutilizan recursos naturales y aquellos que son determinantes de riesgos ambientales.

- Identificar estrategias globales y regionales que permitan un uso sostenible de los recursos y la prevención de los riesgos ambientales.

En esta ocasión debemos denunciar, lamentablemente, que esas expectativas han desaparecido y por ello, de no adoptarse medidas correctivas y esclarecedoras, se estará consumando un retroceso que atenta contra la Formación Ciudadana de los egresados de la ESOR.

De un modo similar, recientemente se promulgó Resolución del CFE (Res CFE 180-12, del 26-092012), referida a la Formación General del Ciclo Orientado de la ESOR y en su Art $1^{\circ}$ se enumeran explícitamente las disciplinas que, a criterio del CFE, integran dicho ciclo. En efecto, el Anexo 02 de dicha resolución (Res CFE 180-12 (02), que se refiere a los NAP del campo de las Ciencias Naturales, sólo alude a las disciplinas Biología, Física y Química

Como ya se ha señalado, estas omisiones constituyen un grave error epistemológico y pedagógico e, inevitablemente, promueve una enseñanza deformada y una visión distorsionada de la comprensión sistémica de la naturaleza (Lacreu, 1999 p.244). De este modo se han suprimido tanto los saberes geológicos (conceptuales y metodológicos) como la caracterización de los objetos de estudio que son propios, exclusivos y excluyentes del campo de las Ciencias Naturales.

Además, es necesario destacar la gravedad del problema que señalamos: la omisión de dichos saberes impide una adecuada Formación Ciudadana (Lacreu, 2009) y por ende se propicia el analfabetismo geológico. Consecuentemente se dificulta a los sujetos políticos reflexionar, sostener miradas críticas y argumentar adecuadamente acerca de las problemáticas ambientales referidas al manejo inadecuado de los recursos y riesgos geológicos. Esta situación indeseada, es el resultado de decisiones políticas (Lacreu, 2012) adoptadas por acción o por omisión y, oportunamente, habrá que deslindar las responsabilidades de los sujetos e instituciones involucradas (nacionales, provinciales y universitarias).

\section{Falacias o inconsistencias propositivas}

Muchos de los loables propósitos incluidos en los "Lineamientos Políticos y Estratégicos de la Educación Secundaria Obligatoria", serán de cumplimiento imposible sin la contribución de los saberes de la geología. De allí que caractericemos dos falacias del tipo "Non Sequitur" (García Damborenea, 2000) ya que se han detectado inconsistencias evidentes entre las finalidades que se proponen y los medios previstos para alcanzarlas. Seguidamente se analizan dos casos:

Falacia 1: A propósito de los saberes a enseñar en los ciclos "Básico" y "Orientado" de la Educación Secundaria Orientada (Res 84-09 -82-), se señala que ".... en dichos ciclos, recuperan los campos de formación de presencia generalizada en los diseños curriculares jurisdiccionales vigentes, y que dotan a las ofertas de un equilibrio acorde con las finalidades de la escolaridad secundaria,..."

La redacción de dicho apartado es poco feliz ya que no se percibe la citada "recuperación". Muy por el contrario, como se mencionó anteriormente, se han ocultado los antecedentes constituidos por los CBC (Cs Naturales) de la ex Educación Polimodal (Lacreu, 2007 a, 2009) específicamente aquellos correspondientes al Bloque 4: "Los subsistemas 
terrestres: recursos naturales y riesgos ambientales" que, aunque insuficientes, podrían justificar mínimamente la afirmación: “...dotan a las ofertas de un equilibrio acorde con las finalidades de la escolaridad secundaria...". Por cierto, el ocultamiento curricular de dichos CBC entorpece la pregonada, necesaria y saludable Formación Ciudadana.

En este contexto la expresión "ocultamiento curricular", en realidad se corresponde con el denominado currículum nulo (Flinders et al., 1986), por cuanto dichas prescripciones, por diferentes motivos, no forman parte de las enseñanzas que efectivamente reciben los alumnos (Sellés-Martínez, 2013 p.4). Esta realidad, en lugar de ser modificada en beneficio de la formación de sujetos políticos es dócilmente aceptada suprimiéndose, en la práctica, los contenidos del citado Bloque 4.

Más adelante se profundizará la argumentación sobre las consecuencias de tal ocultamiento pero, sobre la base de lo expuesto y considerando la ausencia de la enseñanza de las geociencias, cabe preguntarse:

¿Qué formación poseerá un Bachiller, egresado de la Educación Secundaria Obligatoria Argentina, para comprender, reflexionar y actuar políticamente en defensa de una buena calidad de vida, si ignora las razones, procesos y transformaciones geológicas que permiten explicar y predecir la ocurrencia y/o distribución de Recursos Naturales (minería, suelos, agua, territorios), y su vulnerabilidad respecto de las acciones humanas, o de los Riesgos Naturales (volcanismo, terremotos, inundaciones, avalanchas, etc.)?

Esta circunstancia constituye una falacia por cuanto denota la inconsistencia lógica, pedagógica y epistemológica entre el propósito enunciado sobre la Formación Ciudadana y los medios educativos que el CFE propone para alcanzarla. La importancia de las Geociencias en la Formación Ciudadana se puede consultar en Lacreu, H.L. (2009) ya que en este documento se priorizará el análisis de una segunda Falacia.

Falacia 2: Del análisis del apartado 87 de la citada Res 84-09 surgen nuevas inconsistencias. En él se señala que todos los estudiantes Educación Secundaria Obligatoria “...accederán a una formación que: ... d) incluya los saberes de diversas Ciencias Naturales en ambos ciclos, de modo que los estudiantes de cualquier oferta del nivel se encuentren preparados para 1: interactuar con los fenómenos naturales y 2: profundizar en la construcción de los modelos explicativos básicos de las ciencias; para 3: comprender el mundo natural y su funcionamiento y para 3: tender puentes entre los saberes cotidianos y los científicos, a partir de propuestas de enseñanza que recuperen sus propias preguntas, inquietudes y explicaciones. Para ello será necesario considerar los aportes específicos de la Física, la Química y la Biología" (números e itálica son del autor)

El concepto precedente, señalado con letras en itálica, no podría ser más desafortunado ya que se vuelve a poner en evidencia la concepción errónea sobre la delimitación del campo epistemológico de las Ciencias Naturales en donde se omiten los aportes de la geología. También se percibe la ausencia de la astronomía pero, en la práctica ésta podría formar parte de los contenidos de la física (quizás, como física teórica). En cambio, los contenidos y procedimientos de las Ciencias Geológicas poseen una identidad y singularidad tan específicas, que resultan imposibles de subsumir en ninguna otra disciplina.

Dicha concepción epistemológica ha influido negativamente en la propuesta pedagógica y en los diseños curriculares jurisdiccionales que de ella se derivan. En todos los casos, nuevamente se muestra la falta de consistencia entre lo que se espera lograr y los medios que se proponen para hacerlo.

Se puede apreciar que en el mencionado apartado 87 se proponen cuatro metas, o competencias, imposibles de alcanzar si no se incluyen la geociencias en la Formación General de la Educación Secundaria, a saber:

1-"interactuar con los fenómenos naturales"

2-"profundizar en la construcción de los modelos explicativos básicos de las ciencias"

3-"comprender el mundo natural y su funcionamiento"

4-"tender puentes entre los saberes cotidianos y los científicos"

El texto de dicho apartado constituye una nueva falacia ya que enuncia metas imposibles de alcanzar considerando sólo los aportes de ciertas disciplinas, que resultan insuficientes para lograrlas. En consecuencia, debemos concluir que tal propuesta es inaceptable en una norma pedagógica nacional referida a las Ciencias Naturales y nos preguntamos:

¿Acaso los autores y firmantes de dicha Resolución creen por ventura que los ciudadanos, sin los aportes de las geociencias, podrán reflexionar y actuar conforme a lo enunciado en las cuatro competencias antes señaladas?

Obviamente, ello no podrá ocurrir, y en el 
caso de no incorporar los aportes específicos de la Geología, además del déficit en la Formación Ciudadana y la Educación Ambiental, se perpetuará la actual enseñanza distorsionada de las Ciencias Naturales. En efecto, uno de los resultados será que los futuros ciudadanos carecerán de adecuada formación acerca de los conceptos, métodos de análisis y argumentos para expresar sus acuerdos o desacuerdos en relación con las tensiones derivadas de las políticas ambientales provinciales, nacionales y globales. Dichas tensiones, quedan reflejadas principalmente por el tipo de monitoreo sobre el manejo de los recursos naturales, las imprevisiones referidas a la prevención y posibilidades de predicción de los riesgos geológicos que degradan el ambiente.

La actual propuesta que se analiza, suprime de la Educación Secundaria la enseñanza tanto de los fenómenos antes mencionados como de los modelos sobre el funcionamiento del planeta, qué sólo pueden comprenderse significativamente en el marco de las Geociencias. Cabe agregar que permanecerán vigentes los saberes cotidianos erróneos sobre recursos y riesgos geológicos, sobre los orígenes de las montañas o las formas de circulación y contaminación de las aguas subterráneas, para citar unos pocos ejemplos.

Para facilitar la comprensión de las afirmaciones precedentes, Lacreu (2010) elaboró un documento ${ }^{5}$ que incluyó la Tabla 1 , a fin de poner en evidencia los "asuntos" que quedarían omitidos y "deformados", sin el aporte conceptual, metodológico y sin los trabajos de campo que deberían ofrecerse en la enseñanza de la Geociencias.

\section{Conclusiones y propuestas}

Se advierte la gravedad y profundidad de la crisis por la que atraviesa la enseñanza de las ciencias en general y de las Geociencias en particular. Por ello, y en el convencimiento de que los resultados sólo se obtendrán a mediano y largo plazo, se concluye en solicitar la inmediata adopción de decisiones políticas correctivas para que todos los egresados de la Educación Secundaria Obligatoria de cualquier orientación y modalidad, se constituyan en ciudadanos críticos, capaces de:

5 Documento entregado en mano al Director Nacional de Educación Secundaria: Guillermo Golzman en 2011, y distribuido entre los compañeros del Equipo Federal que elaboró los borradores del Marco de Referencia para la Orientación en Ciencias Naturales, durante las reuniones realizadas en Huerta Grande (2010) y La Pampa (2011). "interactuar con los fenómenos naturales y profundizar en la construcción de los modelos explicativos básicos de las ciencias; para comprender el mundo natural y su funcionamiento y para tender puentes entre los saberes cotidianos y los científicos, a partir de propuestas de enseñanza que recuperen sus propias preguntas, inquietudes y explicaciones. (Res CFE 084, p. 87)

Hasta el presente, sólo se ha podido advertir la incapacidad de las autoridades del Ministerio de Educación Nacional (MEN) para comprender y asumir las responsabilidades que le competen para evitar las distorsiones y falacias ya mencionadas. Como resultado de ello, no es previsible que el MEN sea capaz "per se", de rectificar y orientar adecuada y rápidamente los problemas mencionados.

En efecto, la complejidad y la naturaleza sistémica de los problemas a resolver, requiere del aporte de expertos en enseñanza de las Geociencias que, por razones históricas, no abundan en la Argentina y los que aún permanecen activos, lo hacen únicamente desde el ámbito universitario. En efecto, desde dicho ámbito, pese a las dificultades disciplinarias internas, ha sido posible el surgimiento de aportes y propuestas evidenciadas en publicaciones nacionales e internacionales. Estos aportes pueden verificarse en los trabajos citados en esta contribución y, sobre todo, en la bibliografía incluida en dichos trabajos.

Por tales motivos, a menos que el MEN decida crear equipos propios, se considera necesario apelar a la responsabilidad política, social y científica del ámbito universitario a fin de aportar propuestas y ofrecer colaboración para resolver los problemas mencionados con todos los desafíos que ello implica.

En ese sentido, cabe señalar que desde la AFAG (Asociación de Facultades con carreras de Geología de la República Argentina) se están realizando gestiones para delinear un plan de acción nacional, con alternativas conducentes al mejoramiento de la enseñanza de las Geociencias en la República Argentina. Se prevé que ello será posible a través de canales institucionales tales como el CUCEN (Consejo Universitario de Ciencias Exactas y Naturales) y el CIN (Consejo Interuniversitario Nacional).

A modo de propuesta, se sugiere que entre otros temas, se construya una agenda que considere las siguientes sugerencias y peticiones ante el Ministerio de Educación de la Nación:

1. Designación de geólogos para su capacitación 
Tabla 1. Competencias ausentes en la Formación Ciudadana de los alumnos del nivel secundario por omisión de las Geociencias en los NAP de Ciencias Naturales de la Formación General de ambos ciclos (Básico y Orientado)

\begin{tabular}{|c|c|}
\hline $\begin{array}{l}\text { Competencias } \\
\text { propuestas }(87)\end{array}$ & $\begin{array}{l}\text { Competencias }{ }^{(1)} \text { ausentes sin los contenidos de las Ciencias de la Tierra en la } \\
\text { ESO }\end{array}$ \\
\hline $\begin{array}{l}\text { 1- "interactuar con } \\
\text { los fenómenos } \\
\text { naturales" }\end{array}$ & $\begin{array}{l}\text { Se carecerá de la comprensión de las causas (prevención y soluciones) sobre fenómenos } \\
\text { naturales que ponen en riesgo a las comunidades tales como: terremotos, volcanes, } \\
\text { inundaciones, avalanchas, colapsos, erosión de suelos, contaminación de suelos y } \\
\text { acuíferos, ocupación indebida de territorios, etc. La omisión, ayudará a mantener } \\
\text { los mitos, ideas erróneas y dogmas existentes sobre los fenómenos mencionados } \\
\text { así como respecto del origen y distribución de los recursos naturales no renovables. } \\
\text { Entre otros aspectos, también se debe acotar que una de las formas más significativas } \\
\text { para "interactuar con los fenómenos naturales" es precisamente tomar contacto con } \\
\text { el entorno natural, en toda su complejidad y comprendiendo el origen de las actuales } \\
\text { interacciones sistémicas vivo - no vivo }\end{array}$ \\
\hline $\begin{array}{l}\text { 2- "profundizar en la } \\
\text { construcción de los } \\
\text { modelos explicativos } \\
\text { básicos de las } \\
\text { ciencias" }\end{array}$ & $\begin{array}{l}\text { Estará ausente la comprensión elemental del modelo de la Tectónica de Placas para } \\
\text { explicar/se el funcionamiento del Planeta en general y para hacerlo en temáticas } \\
\text { singulares como las variaciones de biodiversidad en la tierra, las extinciones masivas y } \\
\text { los cambios que ocurrirán en el futuro. } \\
\text { Se obstaculizará la comprensión de la construcción y evolución de los modelos que } \\
\text { permiten conocer la estructura interna de la Tierra, los modelos que explican el origen } \\
\text { y distribución de los recursos mineros, riesgos geológicos (volcánicos - sísmicos) ni las } \\
\text { razones, métodos y criterios para la búsqueda de los primeros y predicción - prevención } \\
\text { de los segundos. }\end{array}$ \\
\hline $\begin{array}{l}\text { 3- "comprender el } \\
\text { mundo natural y su } \\
\text { funcionamiento" }\end{array}$ & $\begin{array}{l}\text { El mundo natural viene evolucionando desde hace } 4800 \text { millones de años y la } \\
\text { aparición de la vida y del hombre provocaron y provocan cambios que sólo se pueden } \\
\text { comprender (y valorar ) tomando como referencia lo que hubiese ocurrido en un } \\
\text { mundo sin su presencia } \\
\text { Así, con el ocultamiento de las Geociencias, se les impedirá una comprensión básica y } \\
\text { significativa de los principios fundamentales y las metodologías históricas para conocer } \\
\text { el pasado geológico. Así se privará a los alumnos de la única disciplina científica que } \\
\text { les permitirá reconstruir la Historia Geológica de su terruño, que es donde desarrollan } \\
\text { su identidad. Estas carencias acerca de la raíz histórica de la Geología dificultará la } \\
\text { participación responsable en las Auditorías Públicas. Estas, obligadamente, deben } \\
\text { realizarse para que la comunidad se exprese sobre los Estudios de Impacto Ambiental, } \\
\text { cada vez que una obra civil (minera, hídrica, vial, agropecuaria, etc.) interfiera con el } \\
\text { funcionamiento del mundo natural. }\end{array}$ \\
\hline $\begin{array}{l}\text { 4- "tender puentes } \\
\text { entre los saberes } \\
\text { cotidianos y los } \\
\text { científicos" }\end{array}$ & $\begin{array}{l}\text { Permanecerán dogmáticamente las ideas populares erróneas que relacionan el clima o el } \\
\text { volcanismo con los terremotos. La idea errónea de atacar la actividad minera, incluso a } \\
\text { la minería "limpia” sin la cual debería paralizarse toda la industria mundial de artefactos } \\
\text { que usan RRNN. De igual modo, se facilitará la proliferación de mitos sobre los ríos } \\
\text { subterráneos, la concepción mágica sobre la permanencia del caudal de los ríos pese a } \\
\text { las sequías y el desconocimiento sobre los procesos íntimos sobre el movimiento de las } \\
\text { aguas subterránea y los perjuicios causados por la sobreexplotación. }\end{array}$ \\
\hline
\end{tabular}

(1) Por competencia se entiende la capacidad de emplear conceptos básicos, de modo oportuno, pertinente, con solidaridad y responsabilidad social. Ello requiere de aprendizajes críticos y significativos, muy diferentes de los aprendizajes mecánicos o memorísticos.

y actuación en los Equipos Curriculares de la educación primaria, secundaria y la formación docente.

2. Rectificación de la Res CFE 84-09 en sus apartados 86 y 87 (d), para incluir a la Geología como disciplina integrante de las Cs Naturales.

3. Rectificación de la Res CFE 180-12 en su 6to considerando y en Art $1^{\circ}$ para la inclusión de la Geología como disciplina integrante de las Cs Naturales.

4. Rectificación de la Res CFE 180-12 (Anexo 02) con el objetivo de incluir el título "Geología" y los correspondientes Núcleos de Aprendizajes 
Prioritarios que se decidan.

5. Ofrecer la colaboración del CUCEN, a través de la AFAG, para participar activamente en la elaboración, organización y desarrollo de la "Estrategia Nacional de mejora en la Enseñanza de las Ciencias Naturales y Matemática" conforme a la Res 186/12 (01-11-12)

6. Sugerir se tengan en consideración los aportes de:

6.a) Comisión Nacional para el Mejoramiento de la Enseñanza de las Ciencias Naturales y la Matemática (Ver en: Comisión Nacional, 2007)

6.b) Aportes a la enseñanza de las ciencias naturales. Documento de especialistas elaborado en el Congreso Internacional de la Enseñanza de las Cs. Naturales y la Matemática. (Ver Aportes de Especialistas, 2008.)

7. Promover la modificación de las normativas de los Profesorados de Enseñanza Primaria y de Biología del Secundario para fortalecer la formación Geológica de sus egresados.

8. Gestionar financiamiento para un programa nacional de Posgrado Universitario en Enseñanza de las Geología para docentes de los niveles primario, secundario y terciario.

Finalmente y considerando las características federales del sistema educativo, se señala la importancia de que cada sede de la AFAG, en su región de influencia, procure articular con el Ministerio de Educación de cada provincia cercana, de un modo similar al expuesto precedentemente.

\section{Bibliografia}

Aportes de Especialistas. 2008. Aportes a la enseñanza de las ciencias naturales. Documento de especialistas elaborado en el Congreso Internacional de la Enseñanza de las Cs. Naturales y la Matemática. (Córdoba. 2008). URL: http:/historiadelaastronomia.files.wordpress.com/2008/12/edu_cong_ int_cien.pdf

Comisión Nacional. 2007. Comisión Nacional para el Mejoramiento de la Enseñanza de las Ciencias Naturales y la Matemática (Ver en: Comisión Nacional 2007). URL: http://coleccion.educ.ar/coleccion/ CD23/contenidos/biblioteca/pdf/doc_comision. pdf

García Damborenea R. 2000. Diccionario de Falacias. URL: http://perso.wanadoo.es/usoderazonweb/ html/conten/arca/dicci/dicci2.htm

Flinders D.J., Noddin N. Thornton S.J. 1986. The Null Curriculum: Its Theoretical Basis and Practical Implications Curriculum Inquiry, 16(1):3342. URL: http://wp.vcu.edu/hhughesdecatur/
files/2013/01/Null-curriculum.pdf

Lacreu H.L. 1996. La geología en la educación Argentina. In Fuentes para la transformación curricular ciencias naturales. Buenos Aires, Argentina: Ministerio de Cultura y Educación de la Nación, p. 179-200.

Lacreu H.L. 1997a. Aportes de las Geociencias a la Formación Ciudadana. San Luis, LAE Revista Alternativas, II(7):63-89. (Serie Espacio Pedagógico). URL: http://academia.edu/3891750/Aportes_de_las_Geociencias_a_la_Formacion_Ciudadañ_Lacreu_HL_1997

Lacreu H.L. 1997b. Las Geociencias en la Alfabetización Científica en: Enseñar Ciencias Naturales, reflexiones $y$ propuestas didácticas. Cap. 7:239-270, Comp: M. Kaufman y L. Fumagalli. México, Ed. Paidos. 270p.

Lacreu H.L. 2009. La importancia de las geociencias para la construcción de ciudadanía en el currículo de la enseñanza básica. en: Sicca N.A.L., Moreira da Costa A.D., Fernández de S.A.S. orgs. Processo Curricular, diferentes dimensões. Florianópolis, Ed. Insular. Cap 1, p. 17-36.

Lacreu H.L. 2010. Reflexiones, fundamentos y propuestas para la incorporación de las Ciencias de la Tierra en la Enseñanza Secundaria Obligatoria. Aporte para los marcos de referencia de la orientación en Ciencias Naturales. Inédito.

Lacreu H.L. 2012. Raíces Políticas del Analfabetismo Geológico. In: Simp. Enseñanza de la Geología, 17, 9-14 julio 2012, Huelva, España. Actas... Huelva, AEPECT. P. 91-99.

Ministerio de Educación, 2008. Cuadernos para el Aula. URL: http://www.me.gov.ar/curriform/cuadernos.html

Res. CFEC 57/97a Contenidos Básicos Comunes para la Educación Polimodal. CBC de Cs Naturales pp. 111 a 133 Ministerio de Cultura y Educación de la Nación, 1997. URL: http://www.me.gov.ar/ consejo/resoluciones/res97/57-97.pdf, y http:// www.me.gov.ar/consejo/documentos/cbc/polimodal/1996/cbccsnat.pdf

Res. CFEC 57/97b Contenidos Básicos Orientados para la Educación Polimodal. CBO de Cs Naturales Ministerio de Cultura y Educación de la Nación, 1997. URL:http://www.me.gov.ar/consejo/resoluciones/ res97/57-97.pdf y http://www.me.gov.ar/consejo/ documentos/cbc/polimodal/cboep/csnat.pdf

Res. CFE 228-04-aneegb Núcleos de Aprendizajes Prioritarios. Primer Ciclo de Egb / Nivel Primario (Cs Nat. 24-28). URL:http://www.me.gov.ar/consejo/ resoluciones/res04/228-04-aneegb.pdf

Res. CFE 235-05 Núcleos de Aprendizajes Prioritarios. Segundo Ciclo de Egb / Nivel Primario. Ciencias Naturales. URL: http://www.me.gov.ar/consejo/ resoluciones/res05/235-05-ane1-cn.pdf

Res. CFE 249-05 Núcleos de Aprendizajes Prioritarios. Tercer Ciclo E.G.B./Nivel Medio. Ciencias 
Naturales (dic. 2005). URL: http://www.me.gov. ar/consejo/resoluciones/res05/249-05-ane1cn.pdf

Res. CFE 84-09. Lineamientos políticos y estratégicos de la educación secundaria obligatoria URL: http://www. me.gov.ar/consejo/resoluciones/res09/84-09-anexo01.pdf

Res CFE 180-12 Núcleos de Aprendizajes Prioritarios del Campo de Formación General. Ciclo Orientado. Educación Secundaria. URL: http://www.me.gov.ar/ consejo/resoluciones/res12/180-12.pdf

Res CFE 180-12 (02) Ciencias Naturales. Núcleos de Aprendizajes Prioritarios del Campo de For- mación General. Ciclo Orientado. Educación Secundaria. URL: http:/www.me.gov.ar/consejo/ resoluciones/res12/180-12_02.pdf

Res. CFE 186-12. Estrategia Nacional de mejora en la Enseñanza de las Ciencias Naturales y Matemática. URL: http://www.me.gov.ar/consejo/resoluciones/res12/186-12.pdf

Sellés-Martínez J. 2013. Informal Educational Strategies in Teaching Geosciences when Formal Courses are Unavailable: The Experience of AulaGEA in Buenos Aires, Argentina. J. Geosc. Educ., 61:3-11 